\title{
Modelling of an uniaxial single-sided magnetically actuated cell-stretching device
}

\author{
Harshad Kamble ${ }^{1}$ Matthew J Barton ${ }^{2,3}$ Nam-Trung Nguyen ${ }^{1}$ * \\ 1 Queensland Micro and Nanotechnology Center, Griffith University, Nathan, QLD, \\ Australia. \\ 2 HealthTech-X, Menzies Health Institute Queensland, Griffith University, Gold Coast, \\ QLD, Australia. \\ 3 Clem Jones Centre for Neurobiology and Stem Cell Research, Griffith University, \\ Nathan QLD, Australia. \\ *Email: nam-trung.nguyen@griffith.edu.au
}

\begin{abstract}
This paper reports the modelling approach and optimization of a magnetically actuated cellstretching device. The paper first describes the numerical simulation of the actuation system consisting of a permanent magnet and an electromagnet. The magnetic flux density and magnetic force were verified experimentally over the range of superimposed magnetic flux density from $186 \mathrm{mT}$ to $204 \mathrm{mT}$. The relative errors for magnetic flux density and magnetic force are 5\% and 15\%, respectively. This systematic modelling approach provides a reasonable numerical model for optimizing the electromagnetic actuator of the cell-stretching device. The induced actuation force was then coupled with the structural analysis of the cellstretching device to determine the acceptable distance between the two magnets. The results suggested that this actuation system is capable of precisely predicting the behavior of our existing cell-stretching device.
\end{abstract}

Keywords: Cell Stretching; Electromagnetic actuator; Modelling; Biomedical device.

\section{Introduction}

Mechanotransduction is the process of converting mechanical stimuli into biological electrochemical signals, which guides cell behavior, growth and morphology of cells [1-4]. Mechanotransduction has been reported as an important factor for maintaining cellular 
homeostasis [5-7]. Therefore, dysfunction or irregularities in cellular mechanotransduction may well result in diseases such as heart failure, asthma and cancer $[8,9]$. For this reason, mechanotransduction has become an increasing interest for researchers in fields such as bioengineering and regenerative medicine. However, controlling the complexities associated with a cell's physical in-vivo microenvironment is a hubris task, which has led to the development of in-vitro devices. In-vitro cell stretching devices are designed to mimic the cells physical microenvironment and provide a greater insight into the complex mechanotransduction mechanism.

Micropipettes or tweezers are two common in-vitro stretching methods used for introducing mechanical force into cells [10-14]. A number of commercial cell-stretching platforms are currently available. For example, Flexcell (Flexcell International Corporation) is considered the most elegant cell-stretching platform in the market. This system incorporates pneumatic actuators to induce a homogenous strain to a membrane, where cells are cultured [15-17]. Strex Systems (STREX Inc.) and ElectroForce (Bose Corporation) are other cell-stretching systems, which also have been widely used [18-21]. Apart from commercial cell-stretching systems, several custom-made cell-stretching devices had been reported in the literature over the last decade [22-26].

Common methods for stretching cells grown on an elastic membrane include: electromagnetic, piezoelectric, optical and pneumatic actuators [22, 27-31]. Ursekar and Teo [25] developed a cell-stretching device with indenter design and utilized a stepper motor to induce homogenous strain onto a thin membrane. Nava, Bragheri [27] introduced optical actuation for cell-stretching purpose. Huang and Nguyen [22] utilized pneumatically actuated multilayerd microfludic device for uniaxial cell stretching. Fior and Maggiolino [24] designed an microelectromechanical systems (MEMS) device with externally controlled piezoelectric actuaion system. Apart from these common methods, dielectrophoresis, electrothermal and electrostatic actuations have also been reported for cell-stretching applications [32-34].

All existing cell-stretching methods have some advantages, but very few devices fulfill the general requirements for robust cell stretching research, such as: precise strain patterning, compatibility with a variety of microscopes and/or imaging systems (for analysis) and high experimental throughput. System modelling and simulation are also imperative, to obtain optimized parametric values in the design and the operation of a cell-stretching devices. System optimization with finite element analysis (FEA) will help to achieve the above requirements and the optimal operation of a cell-stretching device. 
Our previous report on the single-sided uniaxial magnetically actuated cell-stretching system elucidates the design, simulation and characterisation of the developed stretching device platform with preliminary experimental observations of olfactory ensheathing cells in vitro [26]. The previous model incorporates a simple structural model. The present paper focuses on the coupled simulation of an electromagnetic actuator using an electromagnet and a permanent magnet for the uniaxial cell-stretching device. Experimental data subsequently verified the simulation results, which was demonstrated through optimizing the distance between the permanent magnet and the electromagnet.

\section{Device concept}

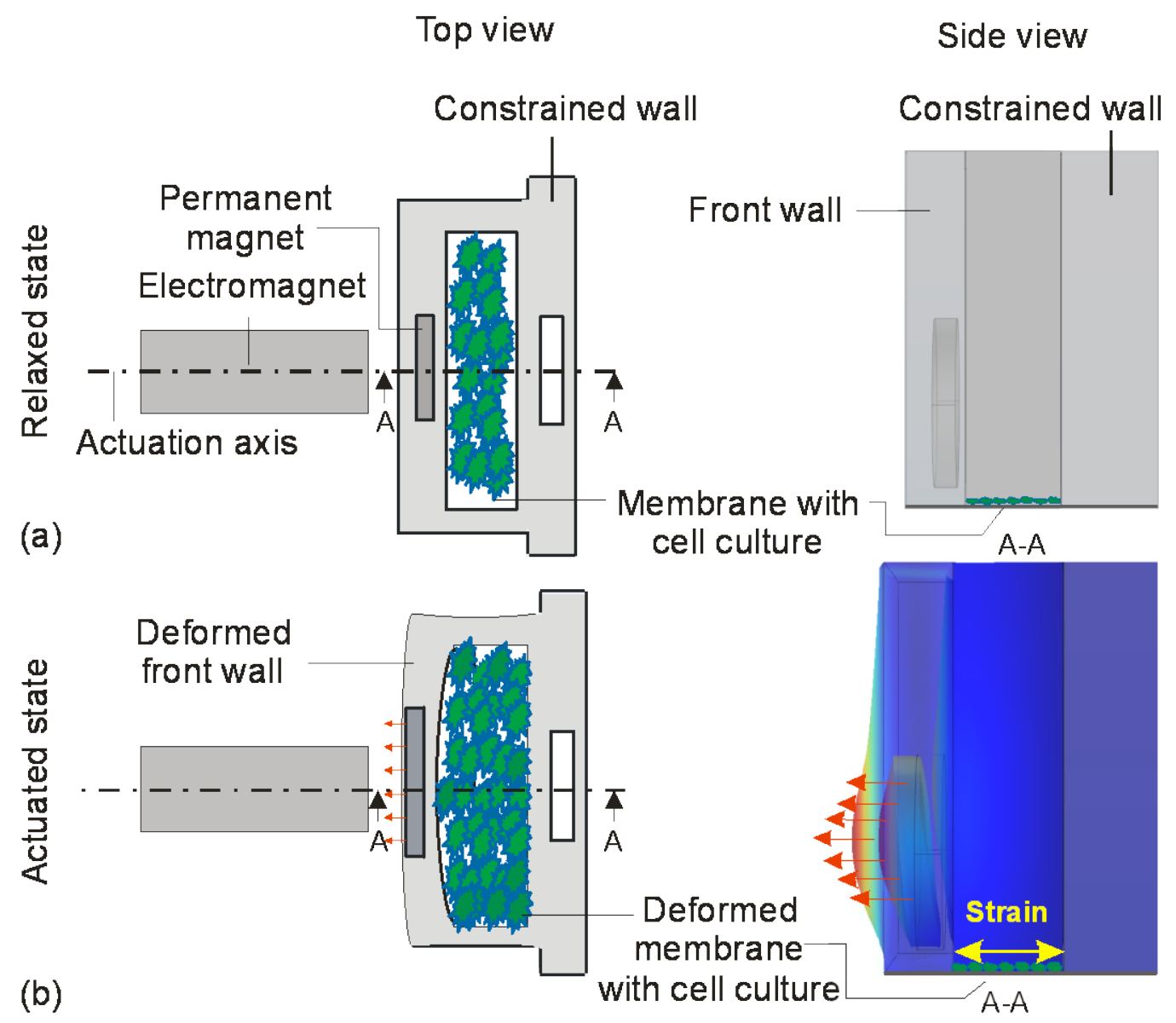

Fig. 1 Operation concept of the cell-stretching device (top view and side view): (a) Relaxed state; (b) Actuated state.

The present numerical study uses the previously reported cell stretching device design as an example, Fig. 1. The cell-stretching system includes a PDMS device and a permanent magnet (PM) with a diameter of $15 \mathrm{~mm}$ and thickness of $2 \mathrm{~mm}$ embedded into the front wall of the device. The permanent magnet is actuated by an electromagnet (EM) controlled by the 
programmable direct-current (DC) power supply. A mounting platform provides a support for the PDMS device and maintains the alignment of the PM and EM along the actuation axis. The 200- $\mu \mathrm{m}$ thick deformable PDMS membrane was bonded to the bottom of the PDMS device using oxygen plasma. Figure 1 illustrates the operation concept of the actuation system. Once the EM is activated, the magnetic force acting on the PM deforms the front wall of the PDMS device, consequently causing the membrane to be stretched uniaxially along the actuation axis.

\section{Modelling approach}

\subsection{Modelling the electromagnet}

The finite element analysis (FEA) model was implemented in COMSOL Multiphysics 5.2 (COMSOL, Inc., MA, USA) utilising AC/DC and structural mechanics modules for the optimisation of our existing cell stretching device [26]. For computational simplicity, the transient nature of the problem is modelled in two quasi-static steps. The first step includes modelling of the actuation system and the calculation of the magnetic force. The second step includes coupling of the magnetic force from the first step with a structural model to determine the strain on the membrane.

The calculation of the magnetic force was further divided into two subtasks. The first task includes modelling the EM and its validation with experimental data to confirm the stability of the model. The second task introduces the PM into the already optimised EM model environment (task 1) to obtain a superimposed actuation condition of the complete cell-stretching device. Once more, experimental data was used to validate the results of the second task.

The linear relationship between the actuating current $I$ and the generated magnetic flux density was used to normalise and to model EM in COMSOL Multiphysics 5.2. Considering the EM as an induction coil in an ideal state and neglecting environmental disturbance the flux density is determined as:

$$
B=\mu N I,
$$

where $B$ is the magnetic flux density (in T), $I$ is the current (in A); $\mu$ and $N$ are the magnetic constant and the number of turns, respectively. 
A static study in AC/DC module with magnetic fields was considered for modelling the EM in COMSOL [35]. The geometry mainly consists of the EM core, the coil and the surrounding medium for magnetic field propagation. The dimension of the EM model was taken from the actual EM [MK magnets, Seoul, Korea] of our existing cell-stretching device. For better visualisation of the simulated magnetic field, a 2D work plane modelling approach was first considered. Subsequently, the $360^{\circ}$ revolution option in COMSOL was utilised to build the 3D EM model with the core and the coil winding. The EM measured $40 \mathrm{~mm}$ in length with a core diameter of $10 \mathrm{~mm}$ and an outer coil diameter of $25 \mathrm{~mm}$ (Fig. 2). The surrounding medium was confined in a cylinder that had a diameter of $15 \mathrm{~mm}$ and length of $52 \mathrm{~mm}$.

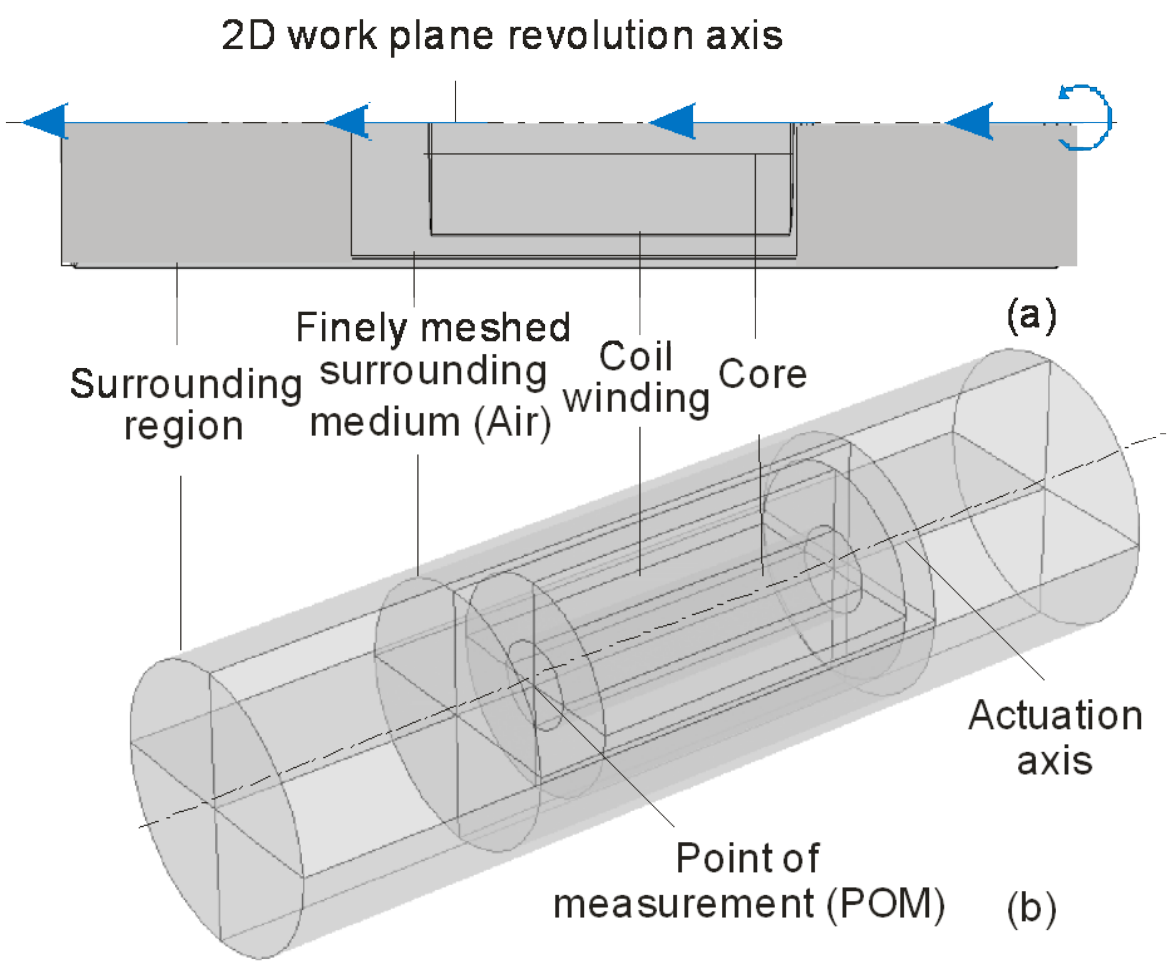

Fig. 2 The geometry of the electromagnet: (a) 2D Work plane; (b) 3D view.

Furthermore, iron, copper and room air were selected to constitute the cylinder core, the coil and the medium domain, respectively. The cylindrical coordinate system was used. Multi turn coil study was selected for the EM modelling assuming the following equation;

$J_{\mathrm{e}}=\left(\frac{N I_{\text {coil }}}{A}\right) e_{\text {coil }}$

where $A$ is total cross section area of the coil domain (in $\mathrm{m}^{2}$ ), $J_{e}$ is current density in the direction of wires (in $\mathrm{A} / \mathrm{m}^{2}$ ) and $e_{\text {coil }}$ is local coil direction. The experimentally obtained coil 
current $I_{\text {coil }}$ (in A), number of turns $N$ and the conductivity of copper of $6 \times 10^{7} \mathrm{~S} / \mathrm{m}$ was considered as input parameters for the model.

The centre of the electromagnet face was considered as the point of measurement to evaluate the magnetic flux density. Following the same experimental setup reported in our previous paper [26], where we obtained the coil current $I_{\text {coil }}$ and the corresponding magnetic flux density $B$ of the EM over the voltage range of $0-15 \mathrm{~V}$. Furthermore, utilizing the parametric sweep option in COMSOL magnetic flux density at the center of the EM along actuation axis was obtained over the above voltage range.

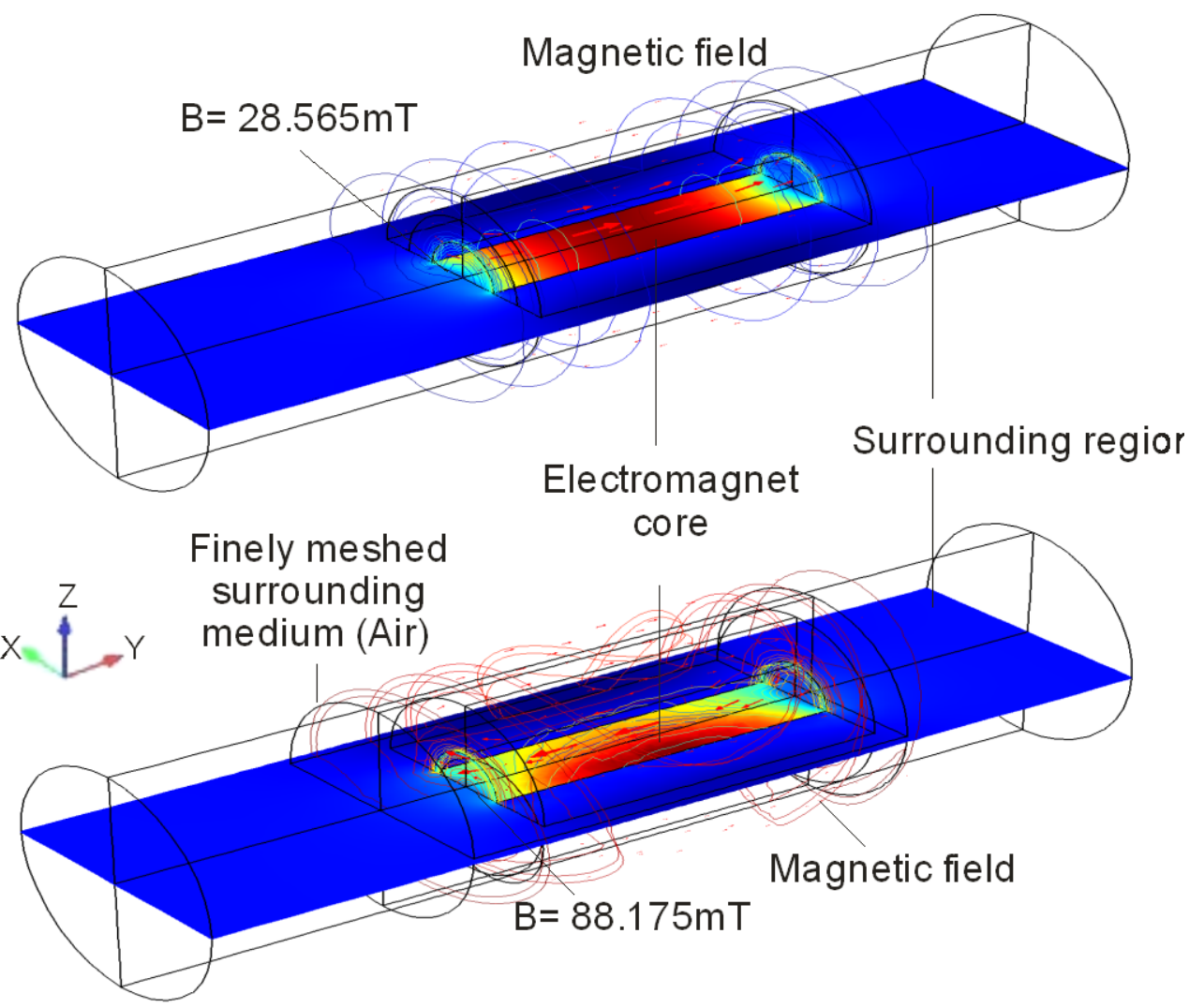

Fig. 3 Magnetic flux density obtained with coil current of $I_{\text {coil }}=0.183 \mathrm{~A}$ : a) With coarse surrounding medium mesh $(\mathrm{B}=28.6 \mathrm{mT})$; b) With optimised mesh of the surrounding $(\mathrm{B}=88.2 \mathrm{mT})$.

The simulation results showed that the magnetic field generated by the EM is heavily affected by the meshing of surrounding medium, Fig. 3. To achieve the optimal modelling conditions, an average of ten magnetic flux density readings (i.e. $B=86.2 \pm 0.6 \mathrm{mT}$ obtained upon actuation of EM with $I_{\text {coil }}=0.183 \mathrm{~A}$ ) was considered as a reference. We observed that the general approach of coarse mesh reduces the effective magnetic field strength (Fig. 3a) yielding a magnetic flux density of only $28.6 \mathrm{mT}$. Thus, a finer surrounding mesh is 
important for accurate propagation of the magnetic field through the surrounding. Moreover, singularities at the edges were required to minimise the errors and obtain realistic simulation. However, a finer surrounding mesh was computationally expensive and adds to the singularities. To mitigate this obstacle, we considered additional smaller medium entities around the EM with finer mesh. Using this optimisation, we observed that finer mesh along the edges and in the immediate environment of the EM improved the wave propagation. This approach greatly reduced the processing time of the COMSOL solver as well as the errors related to wave propagation yielding a magnetic flux density of $88.2 \mathrm{mT}$, Fig. 3b.

Finally, the model was meshed according to the refined geometry domains and obtained a corresponding magnetic flux density at the point of measure (POM) along the actuation axis. The simulation results agreed well with the experimental data with an error variance of $5 \%$, which is also the error of the Gauss meter, Fig.4. The agreement of the data verifies the numerical model of the EM.

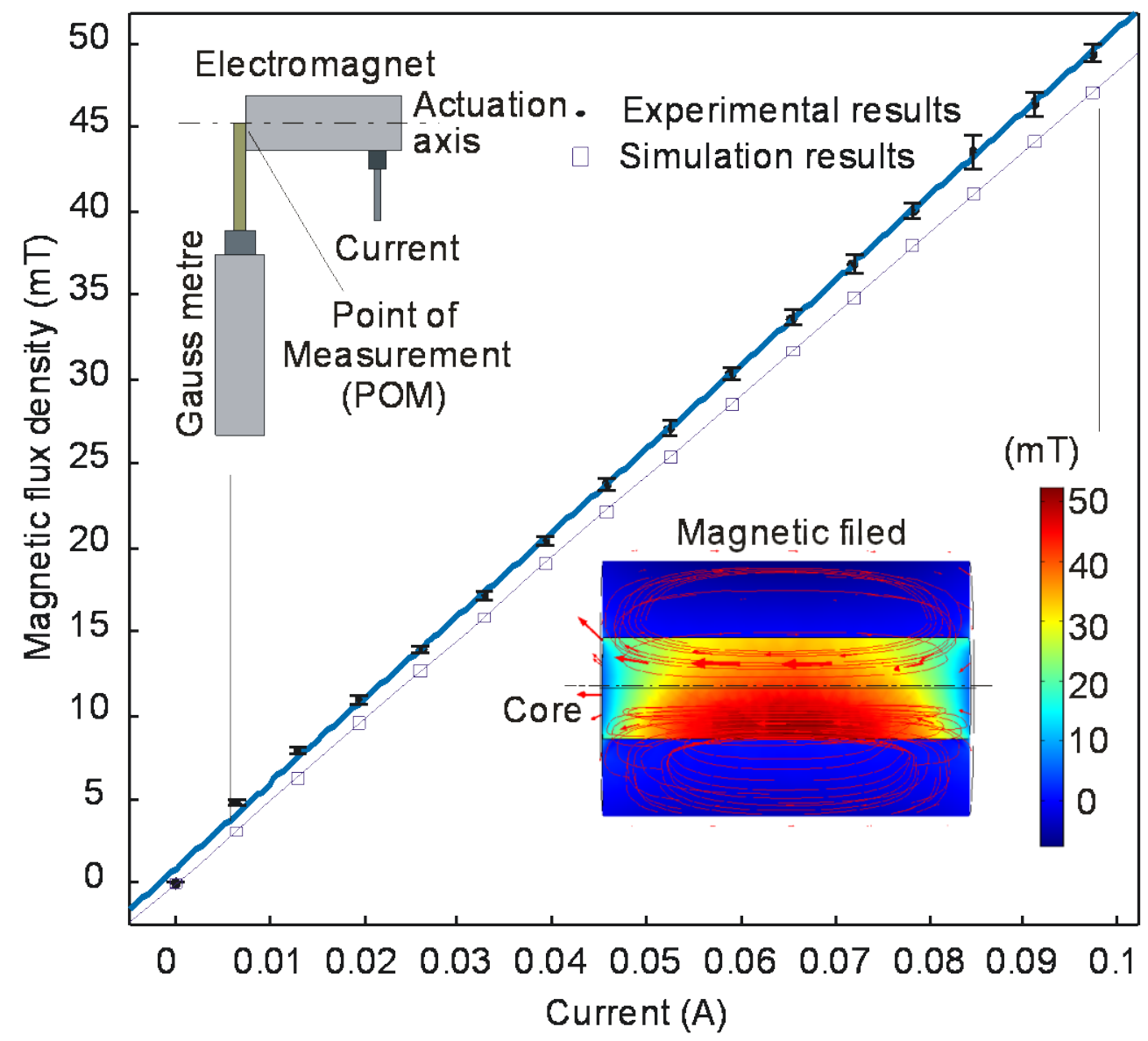

Fig. 4 Magnetic flux density versus applied current at the surface of the electromagnet (inset: experimental setup and COMSOL Simulation result at 15V and 0.0911A.) 


\subsection{Modelling the magnetic force}

To determine the magnetic force, the PM was placed into the previously modeled EM environment (section 3.1). The magnetic fields of the two magnets were superimposed, and the force exerted on the surface of the PM was then determined.

\subsubsection{Modelling the permanent magnet}

The same axial symmetric approach as described in Section 3.1 was utilized to build the PM geometry with a thickness of $2 \mathrm{~mm}$ and a diameter of $7.5 \mathrm{~mm}$. Axially aligned PM was introduced into the system at a distance of $6 \mathrm{~mm}$ away from the EM face along the actuation axis to model the position of the PM and the EM in the actual cell-stretching device. Neodymium (NdFeB) was selected as the material of the permanent magnet.

The Maxwell-Amperes law was utilized to describe the PM:

$$
\nabla \times \mathbf{H}=\mathbf{J}, \mathbf{B}=\nabla \times \mathbf{A} \text {, and } \mathbf{J}=\sigma \mathbf{E} \mathbf{B}
$$

where $\mathbf{H}$ is magnetic field strength $(\mathrm{A} / \mathrm{m}), \mathbf{J}$ is current density $\left(\mathrm{A} / \mathrm{m}^{2}\right), \mathbf{A}$ is magnetic vector potential $(\mathrm{Wb} / \mathrm{m}), \mathbf{E}$ is electric filed $(\mathrm{V} / \mathrm{m})$ and $\mathbf{B}$ is magnetic flux density (T). Further, to incorporate an appropriate input we calculated the magnetic remanence $(\mathrm{Br})$ of the PM. The magnetic flux at the centre surface of the permanent magnet along the actuation was measured with a Gauss meter (Hirst Magnetic Instrument Ltd.). Ten measurements were taken, and the average magnetic flux density was $B=206.2 \mathrm{mT}$. This experimental value was used to calculate the magnetic remanence $(\mathrm{Br})$ of the model according to the relationship:

$B=\frac{B r}{2}\left(\frac{D-z}{\sqrt{R^{2}+(D+z)^{2}}}-\frac{D-z}{\sqrt{R^{2}+(D+z)^{2}}}\right)$

where $D=2 \mathrm{~mm}$ and $R=7.5 \mathrm{~mm}$ are the thickness and the radius of the PM, respectively. The axial distance from the surface was taken as $\mathrm{z}=0 \mathrm{~mm}$.

The obtained magnetic remanence $(B r=1.6 \mathrm{~T})$ served as an input for the model of the PM. Considering the spring constant of the PDMS wall in the actual cell-stretching device, the back surface of the PM was fixed to obtain the force reaction onto the PM upon the field of the EM.

\subsubsection{Modelling the superimposed system with electromagnet and permanent magnet}


For the superimposed condition, the magnetic flux density at the centre and on the surface of the PM was considered. For the measurement, both magnets were mounted $6 \mathrm{~mm}$ apart onto the linear stage of a syringe pump along the actuation axis. The Gauss meter was mounted perpendicular to the auction axis and the magnetic flux density reading where taken at POM over the operational range of $0-15 \mathrm{~V}$.

To obtain optimal modelling conditions, the magnetic flux density was evaluated with the maximum actuation voltage of $15 \mathrm{~V}$ or a corresponding coil current of $I_{\text {coil }}=0.0911 \mathrm{~A}$. Ten measurements resulted in an average magnetic flux density of $\mathrm{B}=209 \pm 0.52 \mathrm{mT}$ which was considered as a benchmark.

To achieve the optimal modelling benchmark, we included an additional finely meshed medium entity in between EM and PM to avoid propagation error in the coupled system, Fig.5. Finally, the system was meshed according to the geometry domain and solved to obtain superimposed magnetic flux density $B$ at the centre of the PM surface over the range of coil current $I_{\text {coil }}$ corresponding to the voltage range of 0-15 V. Figure 6 illustrates the simulation and experimental results of the magnetic field versus the actuation current.

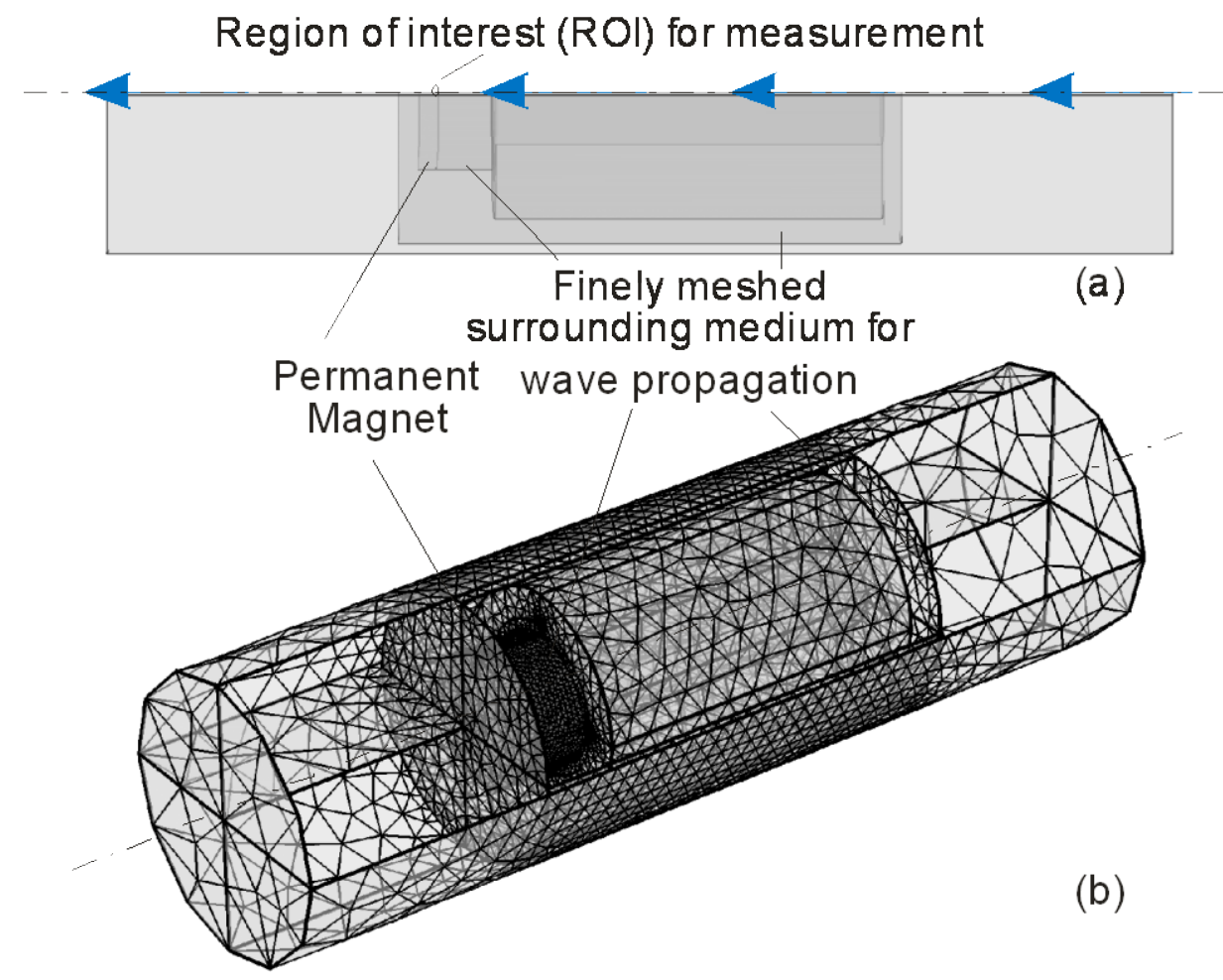

Fig. 5 Meshed model of the two magnets for the simulation of the magnetic force acting on the permanent magnet: a) 2D work plane for coupled geometry; b) Optimised meshing conditions for simulation. 


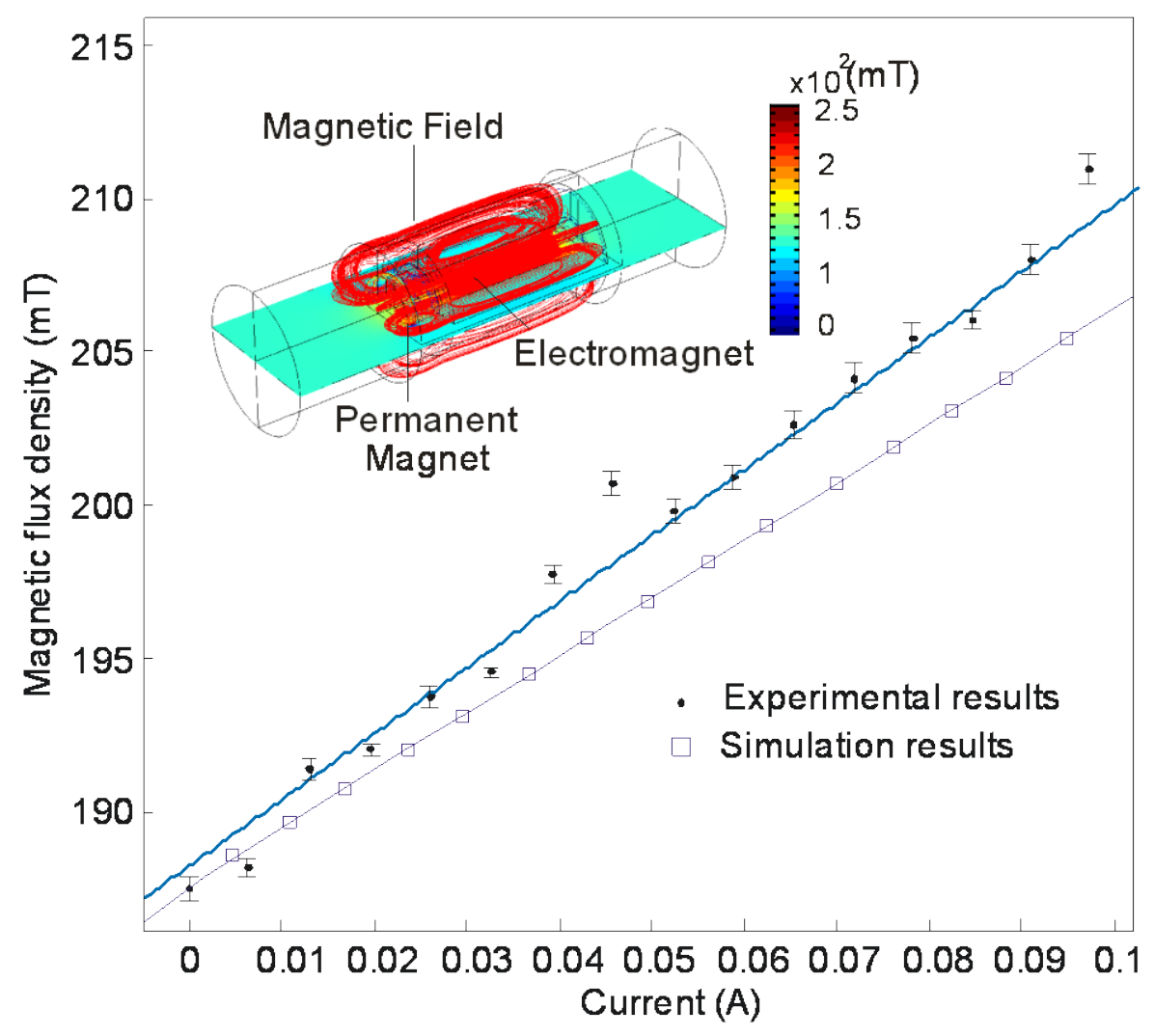

Fig.6 Superimposed magnetic flux density on the surface of the permanent magnet versus the actuation current (Inset: COMSOL simulation result at 15V with 9,11 mA yielding $203.36 \mathrm{mT}$.)

\subsubsection{Magnetic force calculation}

Maintaining the optimized modelling conditions for the superimposed case magnetic force was calculated at the surface of the PM along the actuation axis. Force calculation in the magnetic field study was utilised to obtain the force exerted onto the PM assuming following equation:

$\mathrm{F}=\left[\int_{-\delta ; \Omega} n T d s\right]$

$\tau=\left[\int_{-\delta ; \Omega}\left(\mathrm{r}-\mathrm{r}_{0}\right) \mathrm{X}(\mathrm{nT}) d s\right]$

$\tau_{\mathrm{ax}}=\frac{\mathrm{r}_{\mathrm{ax}}}{\left|\mathrm{r}_{\mathrm{ax}}\right|} \cdot \tau$

where $F$ is total force (in $\mathrm{N}$ ), $\tau$ is torque (in $\mathrm{Nm}$ ), $\mathrm{T}$ is Maxwell stress tensor (in $\mathrm{Pa}$ ), $n$ is outward normal from the domain and $\mathrm{r}_{0}$ is a point on the axis of rotation. 


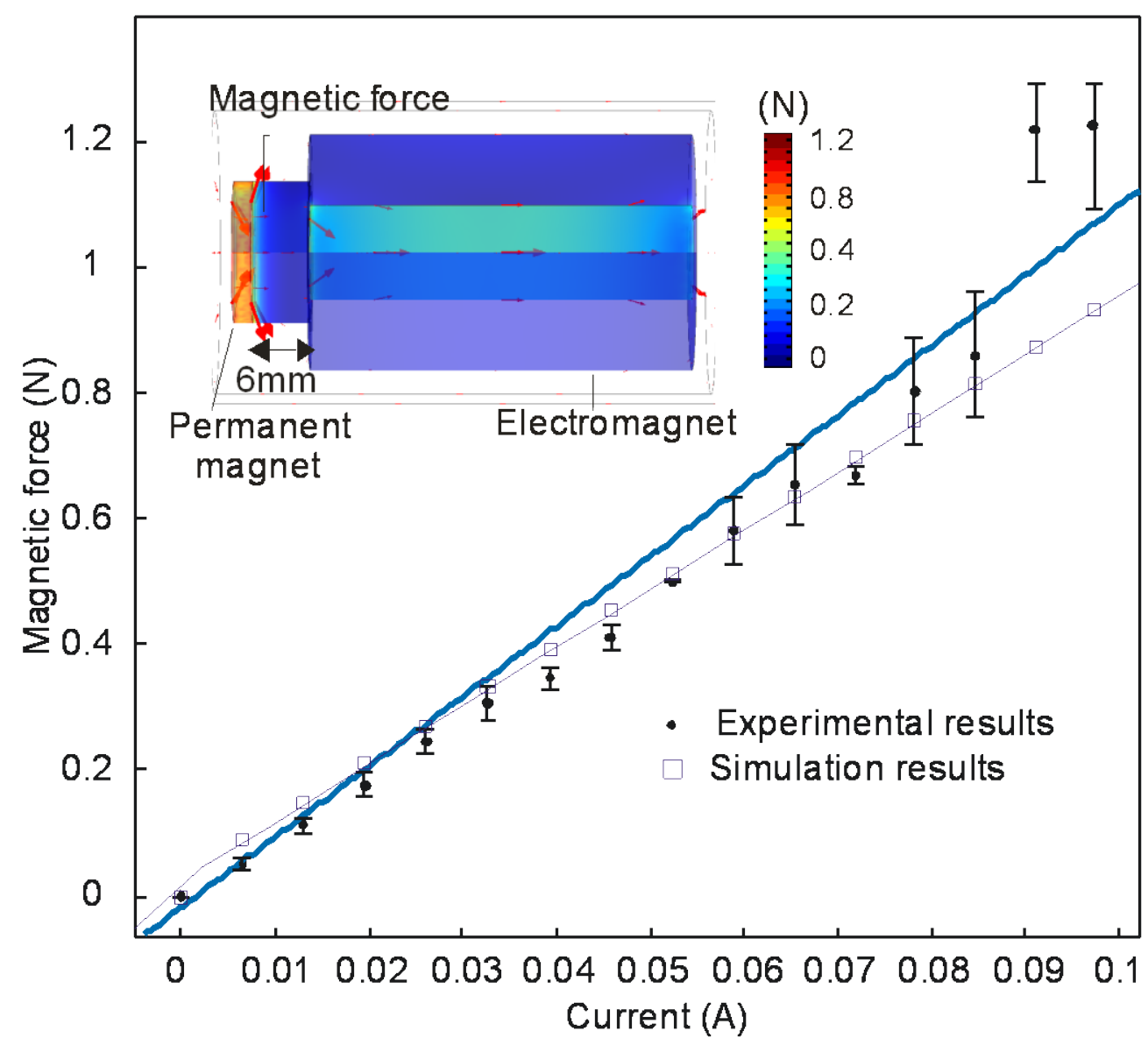

Fig. 7 Magnetic force versus applied current (Inset: COMSOL Simulation result at 15V and 0.973A generating $1.2 \mathrm{~N}$ force on the surface of the PM).

Figure 7 compares the simulated magnetic force with the measured magnetic force. The magnetic force was measured in our previous work by fitting the actual deflection of the cell-stretching device with a FEA model [26]. The statistical analysis of the data provides an error variance of $15 \%$, which is acceptable considering the simplicity of the model and the practical requirement.

\subsection{Modelling the magnetically actuated cell-stretching device}

As the magnetic force acting on PM was modelled and verified in section 3.2, the next step was to couple the magnetic force with the structural model of the cell-stretching device. In our previous work [26], the acceptable working distance between PM and EM was chosen as $5 \mathrm{~mm}$, confirming no visible actuation. As the force requirement varies depending on the cells under investigation. The FEA model described here can be utilized to optimize the acceptable distance for better force stability. We utilised the FEA model and calculated the force exerted on the PM surface at $15 \mathrm{~V}$ or $I_{\text {coil }}=91.1 \mathrm{~mA}$ by keeping the PM fixed and 
varying the EM position over the range of 0.3 to $8 \mathrm{~mm}$ along the actuation axis. Parametric sweep option in COMSOL was chosen to predict the system behavior.

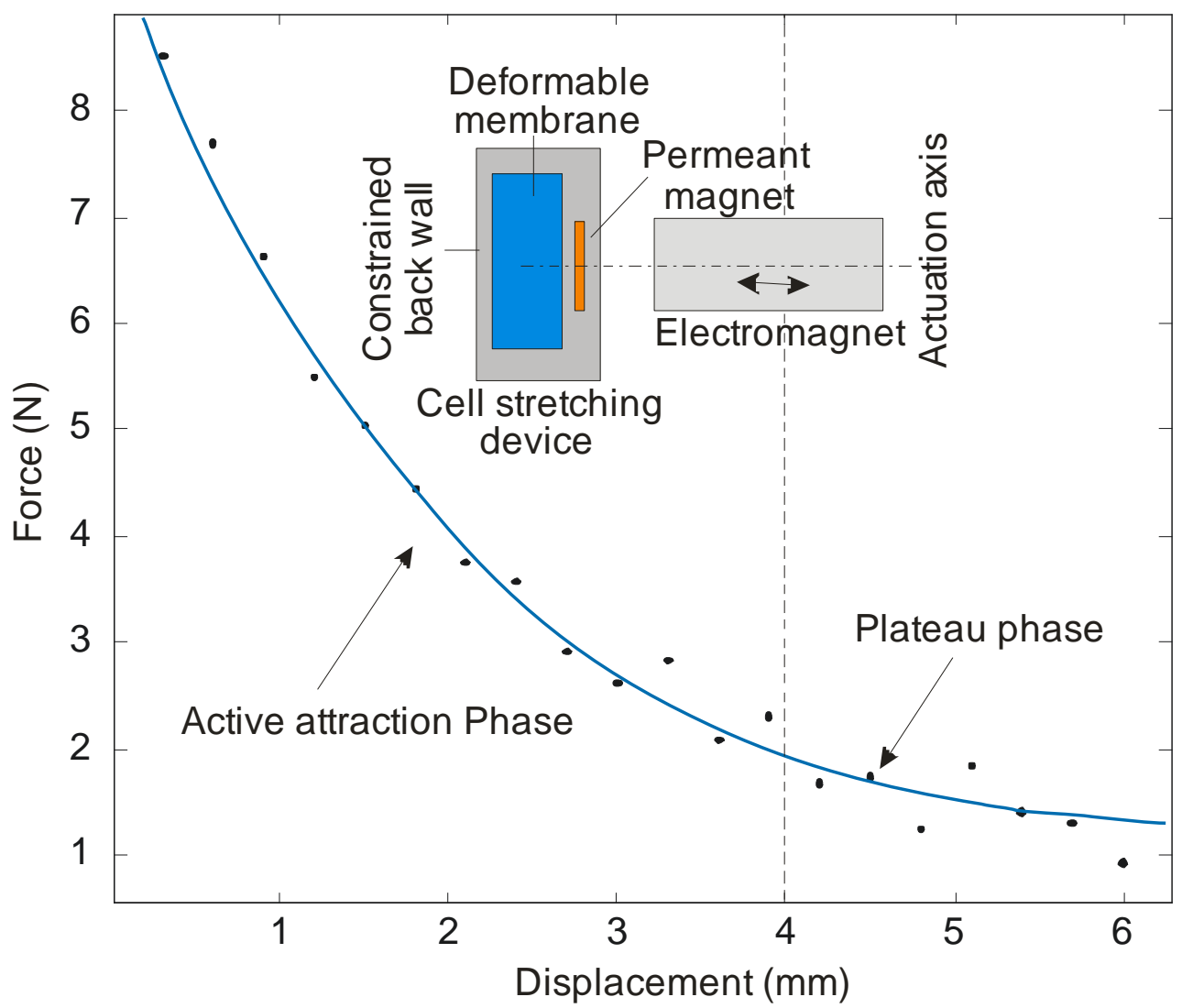

Fig. 8 Simulated actuation force on the permanent magnet at different distances between the two magnet (actuation voltage of $15 \mathrm{~V}$ with the corresponding actuation current of $91.1 \mathrm{~mA}$ ).

Figure 8 depicts the simulation results, indicating the two distinct phases: 1) active attraction phase where the force was observed to decrease exponential from $8 \mathrm{~N}$ at $0.3 \mathrm{~mm}$ to $2.3 \mathrm{~N}$ at $3.7 \mathrm{~mm}$ and 2) the stable phase, where after $4 \mathrm{~mm}$ the average force of $1.451 \mathrm{~N}$ can be observed, with a $6 \%$ variation over the range of 4 to $5.7 \mathrm{~mm}$. Thus, 4.2 to $5.7 \mathrm{~mm}$ are considered as a stable acceptable distance range between the PM and EM, that yielded $1.6 \mathrm{~N}$ and $1.3 \mathrm{~N}$ respectively. These results agree well with the empirical distance of $5 \mathrm{~mm}$ used in our previous cell-stretching experiments [26].

\section{Conclusions}

A magnetically actuated in vitro cell-stretching device that consisted of a permanent magnet and an electromagnet was investigated. The numerical FEA model based on the Maxwell equations were verified with experimental data and agreed well. The error variance of $5 \%$ and $15 \%$ for magnetic flux density and magnetic forces, respectively, were obtained for the numerical model. The model was used for determining the optimal distance between the two 
magnets for controlling the magnetic force acting upon permanent magnet. The actuation force provides a precise control of uniaxial strain introduced onto cells. The presented model is capable of accurately predicting the behaviour of our existing in vitro cell-stretching device.

\section{References}

[1] B.D. Hoffman, J.C. Crocker, Cell Mechanics: Dissecting the Physical Responses of Cells to Force, Annu Rev Biomed Eng, 11(2009) 259-88.

[2] C.L. Happe, A.J. Engler, Mechanical Forces Reshape Differentiation Cues That Guide Cardiomyogenesis, Circ Res, 118(2016) 296-310.

[3] F.H. Silver, L.M. Siperko, Mechanosensing and mechanochemical transduction: how is mechanical energy sensed and converted into chemical energy in an extracellular matrix?, Crit Rev Biomed Eng, 31(2003) 255-331.

[4] R.P. Brandes, N. Weissmann, K. Schroder, Nox Family NADPH Oxidases in MechanoTransduction: Mechanisms and Consequences, Antioxid Redox Signal, 20(2014) 887-98.

[5] D.J. Leong, J.A. Hardin, N.J. Cobelli, H.B. Sun, Mechanotransduction and cartilage integrity, in: M. Zaidi (Ed.) Skeletal Biology and Medicine Ii: Bone and Cartilage Homeostasis and Bone Disease, Blackwell Science Publ, Oxford, 2011, pp. 32-7.

[6] Y. Cui, F.M. Hameed, B. Yang, K. Lee, C.Q. Pan, S. Park, et al., Cyclic stretching of soft substrates induces spreading and growth, Nat Commun, 6(2015) 6333.

[7] J.H. Wang, B.P. Thampatty, An introductory review of cell mechanobiology, Biomech Model Mechanobiol, 5(2006) 1-16.

[8] C.C. DuFort, M.J. Paszek, V.M. Weaver, Balancing forces: architectural control of mechanotransduction, Nat Rev Mol Cell Biol, 12(2011) 308-19.

[9] D.E. Ingber, Mechanobiology and diseases of mechanotransduction, Ann Med, 35(2003) 564-77.

[10] Y.H. Tan, D. Sun, J.Z. Wang, W.H. Huang, Mechanical Characterization of Human Red Blood Cells Under Different Osmotic Conditions by Robotic Manipulation With Optical Tweezers, IEEE Trans Biomed Eng, 57(2010) 1816-25.

[11] S. Nishimura, K. Seo, M. Nagasaki, Y. Hosoya, H. Yamashita, H. Fujita, et al., Responses of single-ventricular myocytes to dynamic axial stretching, Prog Biophys Mol Biol, 97(2008) 282-97. 
[12] N.J. Sniadecki, C.M. Lamb, Y. Liu, C.S. Chen, D.H. Reich, Magnetic microposts for mechanical stimulation of biological cells: Fabrication, characterization, and analysis, Rev Sci Instrum, 79(2008) 044302.

[13] Y.L. Sun, Z.P. Luo, A. Fertala, K.N. An, Stretching type II collagen with optical tweezers, J Biomech, 37(2004) 1665-9.

[14] O. Chaudhuri, S.H. Parekh, W.A. Lam, D.A. Fletcher, Combined atomic force microscopy and side-view optical imaging for mechanical studies of cells, Nat Methods, 6(2009) 383-7.

[15] H. Kufaishi, M. Alarab, H. Drutz, S. Lye, O. Shynlova, Static Mechanical Loading Influences the Expression of Extracellular Matrix and Cell Adhesion Proteins in Vaginal Cells Derived From Premenopausal Women With Severe Pelvic Organ Prolapse, Reprod Sci, 23(2016) 978-92.

[16] P.S. Nayak, Y. Wang, T. Najrana, L.M. Priolo, M. Rios, S.K. Shaw, et al., Mechanotransduction via TRPV4 regulates inflammation and differentiation in fetal mouse distal lung epithelial cells, Respir Res, 16(2015) 60.

[17] S. Dhein, A. Schreiber, S. Steinbach, D. Apel, A. Salameh, F. Schlegel, et al., Mechanical control of cell biology. Effects of cyclic mechanical stretch on cardiomyocyte cellular organization, Prog Biophys Mol Biol, 115(2014) 93-102.

[18] Z. He, R. Potter, X. Li, M. Flessner, Stretch of human mesothelial cells increases cytokine expression, Adv Perit Dial, 28(2012) 2-9.

[19] S. Higgins, J.S. Lee, L. Ha, J.Y. Lim, Inducing Neurite Outgrowth by Mechanical Cell Stretch, Biores Open Access, 2(2013) 212-6.

[20] F.H. Bieler, C.E. Ott, M.S. Thompson, R. Seidel, S. Ahrens, D.R. Epari, et al., Biaxial cell stimulation: A mechanical validation, J Biomech, 42(2009) 1692-6.

[21] A.M. Throm Quinlan, L.N. Sierad, A.K. Capulli, L.E. Firstenberg, K.L. Billiar, Combining Dynamic Stretch and Tunable Stiffness to Probe Cell Mechanobiology In Vitro, PLoS One, 6(2011) e23272.

[22] Y. Huang, N.T. Nguyen, A polymeric cell stretching device for real-time imaging with optical microscopy, Biomed Microdevices, 15(2013) 1043-54.

[23] K.B. Roth, K.B. Neeves, J. Squier, D.W.M. Marr, Imaging of a linear diode bar for an optical cell stretcher, Biomed Opt Express, 6(2015) 807.

[24] R. Fior, S. Maggiolino, M. Lazzarino, O. Sbaizero, A new transparent Bio-MEMS for uni-axial single cell stretching, Microsys Technol, 17(2011) 1581-7. 
[25] C.P. Ursekar, S.K. Teo, H. Hirata, I. Harada, K.H. Chiam, Y. Sawada, Design and construction of an equibiaxial cell stretching system that is improved for biochemical analysis, PLoS One, 9(2014) e90665.

[26] K. Harshad, M. Jun, S. Park, M.J. Barton, R.K. Vadivelu, J. St John, et al., An electromagnetic cell-stretching device for mechanotransduction studies of olfactory ensheathing cells, Biomed Microdevices, 18(2016) 1-10.

[27] G. Nava, F. Bragheri, T. Yang, P. Minzioni, R. Osellame, I. Cristiani, et al., All-silica microfluidic optical stretcher with acoustophoretic prefocusing, Microfluid Nanofluid, 19(2015) 837-44.

[28] Y. Shao, X. Tan, R. Novitski, M. Muqaddam, P. List, L. Williamson, et al., Uniaxial cell stretching device for live-cell imaging of mechanosensitive cellular functions, Rev Sci Instrum, 84(2013) 114304.

[29] D. Tremblay, S. Chagnon-Lessard, M. Mirzaei, A.E. Pelling, M. Godin, A microscale anisotropic biaxial cell stretching device for applications in mechanobiology, Biotechnol Lett, 36(2014) 657-65.

[30] S. Deguchi, S. Kudo, T.S. Matsui, W. Huang, M. Sato, Piezoelectric actuator-based cell microstretch device with real-time imaging capability, AIP adv, 5(2015) 067110.

[31] H. Kamble, M.J. Barton, M. Jun, S. Park, N.-T. Nguyen, Cell stretching devices as research tools: engineering and biological considerations, LChip, (2016).

[32] Y. Iwadate, S. Yumura, Cyclic stretch of the substratum using a shape-memory alloy induces directional migration in Dictyostelium cells, Biotechniques, 47(2009) 757-67.

[33] I. Guido, C. Xiong, M.S. Jaeger, C. Duschl, Microfluidic system for cell mechanics analysis through dielectrophoresis, Microelectron Eng, 97(2012) 379-82.

[34] N. Scuor, P. Gallina, H.V. Panchawagh, R.L. Mahajan, O. Sbaizero, V. Sergo, Design of a novel MEMS platform for the biaxial stimulation of living cells, Biomed Microdevices, 8(2006) 239-46.

[35] C. Multiphysics, User's Guide, version 4.3, COMSOL, Inc., (2012). 